\title{
Hiperatividade Simpática na Obesidade
}

\section{editorial}

\section{José Barreto Campello Carvalheira}

Professor Doutor do Departamento de Clínica Médica, Faculdade de Ciências Médicas, Universidade Estadual de Campinas (Unicamp), Campinas, SP, Brasil.
DTÁ BEM ESTABELECIDO que a obesidade aumenta o risco de insuficiência $\checkmark$ cardíaca e hipertensão arterial $(1,2)$. Certamente a epidemia de obesidade responde por parte do aumento das mortes relacionadas às doenças cardiovasculares observadas no Brasil. Embora a obesidade possa contribuir para a falência cardíaca e a hipertensão, os mecanismos envolvidos são apenas parcialmente conhecidos. Voltera e cols. sugerem que há aumento na atividade simpática em modelo animal de obesidade neuroendócrina (3).

O aumento da atividade simpática é uma característica comum à obesidade em humanos e em modelos animais. O aumento da atividade simpática pode elevar a pressão arterial via vasoconstrição periférica e aumento da reabsorção tubular renal de sódio (4). Como podem ser observados na figura 1, vários fatores estão envolvidos no aumento da atividade simpática associada à obesidade.

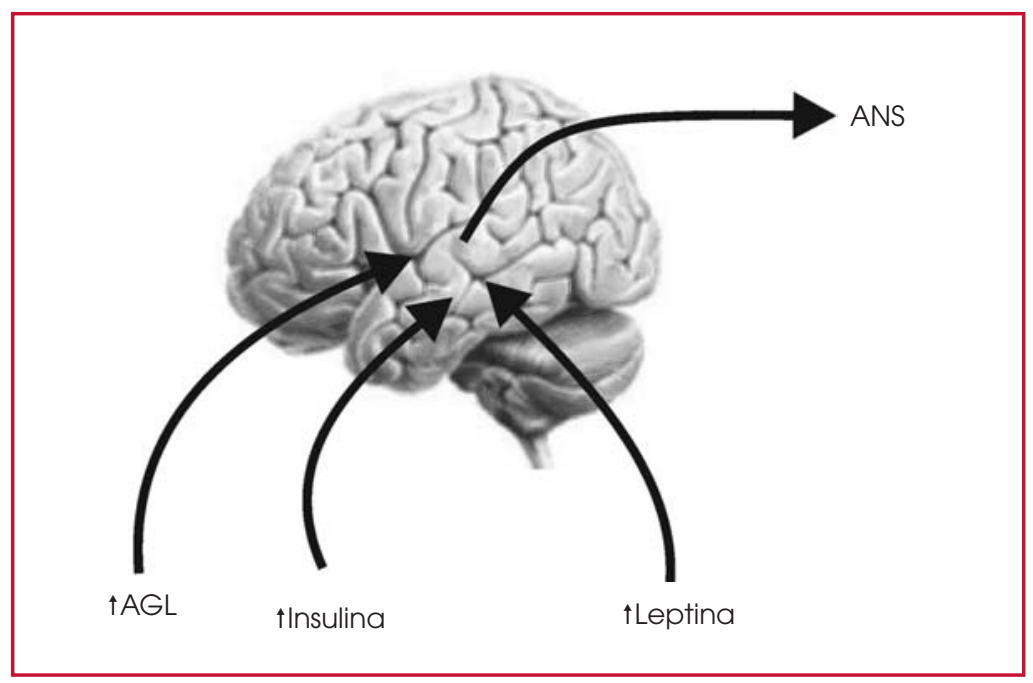

Figura 1. Resumo dos principais mecanismos envolvidos na gênese da hiperatividade simpática na obesidade. $A G L=$ ácidos graxos livres; $A N S$ = atividade nervosa simpática

Evidências recentes indicam que a leptina pode ser o elo entre a obesidade e o aumento da atividade simpática. Além de seus efeitos no controle do apetite e no metabolismo, a leptina age diretamente no hipotálamo para aumentar a atividade simpática $(5,6)$. Entretanto, sabe-se que freqüentemente indivíduos obesos apresentam resistência à ação da leptina no sistema nervoso central e no aumento da atividade simpática simultaneamente. Como conciliar esses dados?

O conceito de resistência seletiva à ação da leptina pode facilitar o entendimento dessa questão no desenvolvimento da hiperatividade simpática em 
obesos. Rahmouni e cols. demonstraram que a leptina em animais obesos preserva sua capacidade de ativar o sistema nevoso simpático direcionado ao rim, enquanto a atividade nervosa simpática dirigida ao tecido adiposo marrom está significativamente reduzida, entretanto os mecanismos que levam a essa divergência de efeitos da leptina ainda permanecem desconhecidos (6).

A hiperinsulinemia também pode participar no desenvolvimento da hiperatividade simpática da obesidade. A insulina, como a leptina, é capaz de aumentar a atividade simpática direcionada aos rins mesmo em animais obesos $(4,5)$. Por outro lado, experimentos que tiveram por fim melhorar a resistência periférica à insulina com aspirina não preveniram o desenvolvimento de hipertensão em cachorros submetidos à dieta hiperlipídica. Assim, o papel da insulina no aumento da atividade simpática permanece controverso (4). Outros mecanismos provavelmente envolvidos na ativação do sistema nervoso simpático na obesidade que devem ser considerados são os altos níveis de ácidos graxos livres circulantes e baixos níveis séricos de grelina e adiponectina (4).

Endocrinologistas, pesquisadores e clínicos passam grande parte de seu tempo pensando em estratégias para melhorar a sobrevida de indivíduos obesos. O estudo de Voltera e cols.(3) deve ajudar a focar a atenção no entendimento da fisiopatologia das doenças car- diovasculares em obesos e no discernimento de qual é a melhor terapia para o tratamento de hipertensão no obeso.

\section{REFERÊNCIAS}

1. Carvalheira JB, Saad MJ. Insulin resistance/hyperinsulinemia associated diseases not included in the metabolic syndrome. Arq Bras Endocrinol Metabol. 2006;50:360-7.

2. Hall JE. The kidney, hypertension, and obesity. Hypertension. 2003;41:625-33.

3. Voltera FV, Cesaretti ML, Ginoza M, Kohlmann Júnior O. Efeito da indução da obesidade neuroendócrina sobre a hemodinâmica sistêmica e a função ventricular esquerda de ratos normotensos. Arq Bras Endocrinol Metab. 2008; 52:47-54.

4. Rahmouni K, Correia ML, Haynes WG, Mark AL. Obesity-associated hypertension: new insights into mechanisms. Hypertension. 2005;45:9-14.

5. Carvalheira JB, Torsoni MA, Ueno M, Amaral ME, Araujo EP, Velloso LA, et al. Cross-talk between the insulin and leptin signaling systems in rat hypothalamus. Obes Res. 2005;13:48-57.

6. Rahmouni K, Morgan DA, Morgan GM, Mark AL, Haynes WG Role of selective leptin resistance in diet-induced obesity hypertension. Diabetes. 2005; 54:2012-8.

\section{Endereço para correspondência:}

José Barreto Campello Carvalheira

Departamento de Clínica Médica, Faculdade de Ciências Médicas, Unicamp

13083-970 Campinas, SP

E-mail: carvalheirajbc@uol.com.br 